\title{
Charisteria Livio Rossetti Oblata
}

[Il quinto secolo. Studi di filosofia antica in onore di Livio Rossetti. A cura di S. Giombini e F. Marcacci, Perugia 2010, ss. 752. ]

SOFIA RANZATO / Pisa /

Non so se questo sia il caso di tutte le raccolte di saggi in onore di uno studioso, ma Il quinto secolo. Studi di filosofia antica in onore di Livio Rossetti, sembra presentarsi come la più autentica risposta al ricco e personale percorso che questo intellettuale ha compiuto fin qua. Già il fatto che questo volume nasca dall'iniziativa di due giovani studiose che hanno così voluto rendere un degno tributo al loro maestro è un primo indizio del successo che Livio Rossetti ha avuto nel suscitare ammirazione e affetto nei suoi allievi. Non si può peraltro dire che la risposta al progetto di Stefania Giombini e Flavia Marcacci non sia stata ampia e partecipata come appare evidente anche solo dalla mole di questo volume e dalla lunga lista di nomi contenuti nella Tabula gratulatoria.

Il prodotto di questo lavoro si presenta, del resto come, un'opera ben costruita in cui evidenti appaiono le corrispondenze tra la biografia intellettuale di Rossetti - brevemente tratteggiata nella prima parte dell'Introduzione - e i numerosi saggi che sono raccolti nel volume.

L'ampio raggio delle ricerche e delle reti di scambio intellettuale - ed umano - che Rossetti ha avviato, è chiaramente espresso dalla complessità del tema che dà il titolo a quest'opera: Il V secolo. La scelta di questo macrocontentitore - nel nostro caso non è tanto significativa per il suo richiamo all'età d'oro della vita politica e culturale della civiltà greca, quanto per il riferimento a un periodo storico in cui venivano alla luce e si sviluppavano molteplici forme di sapere senza che si arrivasse ad una netta distinzione tra loro e tra le attività politiche e sociali ad esse connesse.

Anche le diverse unità tematiche in base a cui sono suddivisi i diversi saggi - Physis, Logos, Ethos, Pathos - ad eccezione dell'ultima - Per L'amico Livio - si riferiscono ad altrettanti concetti chiave della cultura greca intorno a cui la riflessione si è andata svol- 
gendo con particolare vivacità nel V secolo, senza che, peraltro, sia stata raggiunta una formalizzazione teorica di tali nozioni. In una maniera per certi versi affine, nell'attività intellettuale di Livio Rossetti si intersecano tra loro interessi di studio, iniziative di scambio culturale e forme di didattica a vari livelli, difficilmente distinguibili e classificabili tra loro.

Per fare chiarezza, sia sulla vivace esperienza intellettuale di questo studioso, che sull'ampia gamma di temi toccati dagli studi raccolti in questo volume, appare pertanto molto utile l'Introduzione delle curatrici e la bibliografia completa degli scritti di Rossetti presentata nelle pagine immediatamente successive.

La varietà e il gran numero di contributi contenuti nel nostro libro, peraltro, non permette certo di dare conto - anche solo in maniera cursoria - di ciascuno di essi. Sembra preferibile, pertanto, concentrarsi su alcuni temi intorno a cui ha ruotato, in vario modo, l'attività intellettuale di Rossetti e cercare di vedere in che maniera, studiosi più o meno influenzati dal suo pensiero, ne hanno trattato nei saggi a lui offerti in questa raccolta.

Fin dagli anni giovanili, Rossetti ha nutrito un forte interesse per Socrate, per la forma dialogica legata al suo insegnamento e per i Socratici. Questo interesse ha portato alla stesura di un gran numero di contributi - gli ultimi dei quali sono raccolti in Le dialogue socratique, uscito per le Belles Lettres all'inizio del 2011 - e alla promozione di una serie di incontri dedicati alla letteratura socratica antica. Si pensi solo a Socratica 2005 a Senigallia, a Socratica 2008 a Napoli.

A tale interesse risponde, in questo volume, il contributo dell'amico Giuseppe Mazzara (Università degli Studi di Palermo) che, pur condividendo la proposta - sostenuta con forza da Aldo Brancacci - di inserire il pensiero di Antistene, anche in campo retorico, all'interno della tradizione socratica, cerca di valutare, in maniera equilibrata, quale influenza possano avere esercitato sulla sua opera i Pitagorici e, soprattutto, Gorgia.

Tra i saggi raccolti nel Quinto secolo dedicati a Socrate, si distinguono, tra l'altro, da un lato i contributi di Rachel Gazolla (Pontíficia Universidade Católica de São Paulo) e di Gilbert Romeyer Dherbey (Université Paris Sorbonne) che si concentrano sull'attività di Socrate come educatore, dall'altro, quelli che considerano la costruzione del personaggio socratico ad opera di Platone. Si pensi, ad esempio, alla lettura che Thomas Robinson (University of Toronto) fa delle argomentazioni di Socrate sull'immortalità dell'anima nel Fedone e alle interessanti considerazioni di Giovanni Cerri (Università degli Studi Roma Tre) sugli intenti ideologici e propagandistici con cui Platone avrebbe costruito la rappresentazione che Socrate fa della sua ricerca del vero sapiente nell'Apologia.

Un altro tema che ha polarizzato l'attenzione di Rossetti nel corso degli anni è l'interesse per le diverse forme di comunicazione, considerate sia in relazione al mondo antico - come è evidente dai suoi numerosi studi sulla struttura dialogica e sulla retorica che in rapporto ai differenti metodi e strumenti di comunicazione del sapere nel mondo contemporaneo, come dimostra la sua lungimirante consapevolezza - fin dai primi anni novanta - della grande utilità che l'informatica può avere nella trasmissione di conoscen- 
za. Si può peraltro supporre che, proprio l'attenzione verso la situazione comunicativa nel mondo antico e i suoi effetti possa essere stata alla base dell'interesse di Rossetti verso nuove forme di comunicazione nella realtà contemporanea, portandolo a realizzare - tra gli altri suoi numerosi contributi in questo settore - l'edizione dell'Eutifrone di Platone nella forma di ipertesto dialogico-interattivo. A questo proposito, nella sezione finale del Quinto secolo, è possibile leggere l'interessante contributo di Massimo Capponi (Università di Perugia) che mette bene in evidenza l'importante ruolo che l'opera di Rossetti ha ricoperto nella recente storia dell'applicazione degli strumenti informatici alla didattica.

Come viene ben messo in luce da Chiara Chiapperini nel suo contributo al volume, è forse proprio da una riflessione e da una rielaborazione in chiave fortemente personale della maieutica di Socrate che prende forma un progetto come Amica Sofia - divenuta Associazione sociale su base nazionale nel 2008 - che si pone il fine di praticare la filosofia con i bambini e con i ragazzi e di diffondere questa pratica tra docenti e pedagoghi.

All'interno di questa raccolta si trovano, del resto, numerose riflessioni sulle diverse forme del discorso nel mondo antico e sui loro effetti nella elaborazione del pensiero filosofico. Tra queste, basti ricordare il breve saggio di Tomás Calvo-Martínez (Universidad Complutense de Madrid) in cui si sostiene che, nel Fedone, le due concezioni della dialettica come "arte del dialogo" e come "metodo delle ipotesi" non siano contrapposte ma, piuttosto, intrecciate tra loro. Nello stesso ambito si può inserire anche lo studio di Stefania Giombini (Gualdo Tadino, Perugia) e Flavia Marcacci (Pontificia Università Lateranense, Città del Vaticano) sulla posizione tra logica e retorica dell'antilogia. Si pensi, inoltre, all'interessante contributo di Diskin Clay (Duke University, Durham NC) sui vari modi in cui Platone cita, con diverse finalità, i testi a lui precedenti, a volte per fare emergere con poche parole un più ampio contesto, altre per proporre una nuova analisi di un testo famoso, altre ancora dando l'impressione di inventare una citazione o di alterarla deliberatamente. Si tratta di un piccolo saggio di una ricerca di grande interesse, giacché suggerisce nuove vie per comprendere la maniera in cui Platone sfrutta la tradizione poetica nota a lui e al suo pubblico per comunicare il suo nuovo messaggio filosofico. È chiaro, peraltro, che una ricerca di questo tipo può essere applicata anche ad altri autori, portando a risultati altrettanto interessanti. All'interno di una riflessione sulle diverse forme attraverso cui un pensatore tenta di trasmettere il suo messaggio, sembra iscriversi anche il suggestivo confronto che Giovanni Casertano (Università degli Studi di Napoli “Federico II") propone tra il modo in cui il mito del re di Lidia Candaule e del suo usurpatore Gige viene narrato in un passo delle Storie di Erodoto e la maniera in cui questo racconto viene riformulato nel II libro della Repubblica di Platone. Alla diversa costruzione del mito nei due autori, corrisponde un diverso messaggio: mentre nello storico si vuole sottolineare la necessità di "un destino che sovrasta gli attori del dramma umano", nell'opera del filosofo ateniese la necessità non viene dall'esterno, ma dall'interno dell'individuo: si tratta della forza costrittiva delle passioni.

Un'analisi specifica di come il linguaggio visivo del teatro tragico abbia contribuito, nel corso del V secolo, alla formulazione della concezione di anima come mente si può leggere nel contributo di Lidia Palumbo (Università degli Studi di Napoli “Federico II”). 
Nestor Cordero (Université de Rennes 1), per parte sua, si occupa di analizzare il modo in cui Antifonte si proponeva di curare le sofferenze dei suoi pazienti attraverso i discorsi.

Un altro dei poli di interesse intorno a cui ha ruotato l'attività di Rossetti è la polymathia - intesa come varietà e ricchezza di saperi - che caratterizza il pensiero del V secolo e, in particolar modo, quello dei "Presocratici". In questa direzione appare di particolare interesse il contributo di Laura Gemelli Marciano (Universität Zürich) che prende in considerazione come, a cavallo tra il V e il IV secolo a.c., in alcuni trattati tecnici di cucina, agricoltura, e, soprattutto, di medicina, vi sia la tendenza a fondare la propria scienza su dei principi generali, ricavati da altre discipline come la "meteorologia" e i "discorsi sulla natura". A questi trattati fanno da contraltare altri come il "De vetere medicina” o il "de Natura hominis" che intendono, invece, circoscrivere l'ambito della disciplina medica e ancorarlo maggiormente all'esperienza pratica. I promotori delle nuove technai, quindi, da un lato intendono fondare tali discipline su una gamma di competenze estesa ad altri settori conoscitivi, dall'altro, cercano di individuare dei principi che distinguano chiaramente le loro discipline dalle altre. In questo nuovo contesto, pertanto, la figura del polymathes si presenta in forma molto diversa da quella del sapiente di età arcaica ancora in possesso di un sapere assoluto in cui i confini tra le discipline erano fluidi e non ben definiti. Alla figura del medico nel mondo antico e alla sua condizione socialmente fragile è dedicato il bel saggio di Mario Vegetti (Università degli Studi di Pavia). Nell'analisi di questo studioso, se da una parte la sua debolezza istituzionale porta il medico a non raggiungere mai una completa stanzialità e all'impossibilità di riferirsi a una serie di garanzie pubbliche di competenza e di correttezza morale, dall'altra, può essere considerata una delle ragioni della particolare audacia e indipendenza di questi specialisti.

Tra i cosiddetti "Presocratici", il primo ad avere attirato con forza le attenzioni di Rossetti è Eraclito. Proprio a Rossetti si deve, infatti, l'organizzazione - già nel 1981 del primo Symposium Heracliteum a Chieti, cui ha fatto seguito, venticinque anni dopo, grazie all'iniziativa di Enrique Hülsz Piccone, un secondo Symposium Heracliteum a Città del Messico. In consonanza con questo interesse troviamo, nel nostro volume, la ricostruzione dell'esordio del libro di Eraclito - seguita da ampio commento - di uno studioso che ha fatto dell'opera eraclitea la passione di una vita come Serge Mouraviev (Gaillard, Mosca).

In questa sede, del resto, non si può non ricordare il grande contributo che Livio Rossetti ha dato allo sviluppo degli studi su Parmenide e sugli Eleati, anche attraverso l'ideazione di un'iniziativa come "Eleatica" - giunta ormai alla VI edizione - in cui, da alcuni anni si incontrano, nel luogo in cui praticavano il loro sapere Parmenide e Zenone, esperti e appassionati del settore e, grazie alla quale, l'antica città di Elea si arricchisce, ogni anno, di nuovi cittadini onorari. Il primo di questi, Nestor Cordero, ha rivolto un saluto e un caloroso ringraziamento al concittadino Rossetti in uno dei testi conclusivi del nostro volume.

Numerosi, non a caso, sono i contributi di questa raccolta dedicati al pensiero degli Eleati. Tra questi di particolare interesse appare la lettura che Beatriz Bossi (Universidad 
Complutense de Madrid) propone del difficile frammento 16 di Parmenide. Secondo la studiosa, la corrispondenza tra la commistione delle membra corporee e ciò che l'uomo intende, denunciata nei primi versi del frammento, non servirebbe tanto a mettere in evidenza il carattere molteplice e mutevole del pensiero umano - secondo quanto intende Aristotele - quanto piuttosto ad individuare un processo conoscitivo comune ad ogni costituente del reale. Nel fr. 16 verrebbe infatti definito nei termini ingannevoli della doxa uno dei principi fondamentali su cui si fonda l'aletheia: la corrispondenza tra pensare ed essere. È possibile, peraltro, che la rappresentazione dell'attività conoscitiva umana che si legge in B 16, in corrispondenza con lo statuto del discorso che occupa la sezione finale del poema, riprenda sì la struttura del discorso veritiero, ma si fondi totalmente sui principi che costituiscono la realtà molteplice e mutevole della doxa. Una riflessione più generale sull'opera di Parmenide e sulla sua posizione all'interno della tradizione del pensiero arcaico, sorretta da una discussione ben documentata del problema del rapporto tra le diverse parti del poema, si può leggere nel contributo di Dario Zucchello (Liceo classico "A. Volta”, Como).

Per un altro verso, la grande attenzione rivolta da Rossetti all'opera di Platone lo ha spinto ad organizzare, nel 1989, un Symposium Platonicum a Perugia, durante il quale è avvenuta la fondazione dell'International Plato Society (IPS). Il ruolo fondamentale di Rossetti nella realizzazione di questa importante associazione viene espressamente riconosciuto da Thomas Robinson in uno dei contributi della sezione finale di questo volume.

Gli stessi meriti vengono riconosciuti a Rossetti da Christopher Rowe (Durham University) in una nota introduttiva alla sua concisa ma densa analisi del ruolo che il personaggio di Socrate sembra ricoprire nel Politico di Platone, fondata sulla lettura di un passo in cui il giovane interlocutore dello Straniero di Elea suggerisce una certa analogia tra l'arte del governare e il gioco della petteia.

Sul rapporto tra Parmenide e Platone è peraltro possibile leggere, all'interno di questo volume, due interessanti contributi. Franco Ferrari (Università degli studi di Salerno) propone, infatti, un'attenta analisi del modo in cui Platone costruisce il personaggio di Parmenide nell'omonimo dialogo - in particolare nella sezione dedicata alla gymnasia - nel tentativo di individuare le sue corrispondenze con la figura storica dell'Eleate e di comprendere il rapporto che il filosofo ateniese intende stabilire con il pensiero del suo illustre predecessore. Chiara Robbiano (Universiteit Utrecht), per parte sua, tenta di capire perché Platone nella Repubblica suggerisca di attribuire alle rappresentazioni degli dèi presenti nei racconti per bambini, uno dei tratti distintivi dell'essere parmenideo: la sua immutevole immobilità. Al fondamentale valore metafisico ed epistemologico che questo attributo ha in Parmenide si aggiungerebbe nell'opera platonica una valenza morale dipendente dalle nozioni di perfezione e di autosufficienza. La rappresentazione degli dèi come esseri buoni, che non mentono e che non cambiano forma può così fungere da modello per i bambini, futuri guardiani della città.

Uno dei tratti più significativi della vita e dell'attività intellettuale di Rossetti è, peraltro, la sua capacità di stringere rapporti con personalità di diversa età ed esperienza 
e di instaurare scambi e contatti con studiosi e università di diversi paesi. Dell'intenso rapporto di scambio con professori dell'Universidad Nacional Autónoma de México costituisce un'intensa testimonianza il contributo di Gerardo Ramirez Vidal e Omar Álvarez nella sezione conclusiva del volume.

Evidente, peraltro, anche dal buon numero di contributi di studiosi che afferiscono a università sudamericane contenuti nella nostra raccolta, è la stretta collaborazione di Rossetti con diverse istituzioni culturali messicane e brasiliane. Dei rapporti con paesi europei in cui gli studi di filosofia antica non godono di una lunga tradizione costituisce significativa testimonianza l'accorato ringraziamento di Marian Wesoly (Univ Poznan, Polonia) posto a suggello di questo volume.

Che l'ideale di una vita filosofica in comune sia uno dei valori che hanno mosso l'attività di Rossetti viene riconosciuto esplicitamente da Gabrielle Cornelli (Universidade de Brasília) all'inizio di un intervento dedicato alla trattazione sistematica degli elementi che permettono di definire - accogliendo una proposta di Walter Burkert - la comunità pitagorica come una setta. Non a caso, tra questi tratti distintivi, spiccano proprio i valori di koinonía e di philía che Livio Rossetti ha cercato di praticare e di diffondere nel corso di tutto il suo percorso intellettuale. 